\title{
Personalizing Bridging Anticoagulation in Patients with Nonvalvular Atrial Fibrillation-a Microsimulation Analysis
}

\author{
Matthew A. Pappas, MD, MPH' ${ }^{1,2}$, Geoffrey D. Barnes, MD, MSc ${ }^{3}$, and Sandeep Vijan, MD, MS ${ }^{1,2}$ \\ 'Center for Value-Based Care Research, Medicine Institute, Cleveland Clinic, Cleveland, OH, USA; ${ }^{2}$ Department of Hospital Medicine, Medicine \\ Institute, Cleveland Clinic, Cleveland, $\mathrm{OH}, \mathrm{USA} ;{ }^{3}$ Department of Internal Medicine, Frankel Cardiovascular Center, The University of Michigan \\ Health System, Ann Arbor, MI, USA.
}

BACKGROUND: Bridging anticoagulation is commonly prescribed to patients with atrial fibrillation who are initiating warfarin or require interruption of anticoagulation. Current guidelines recommend bridging for patients at high risk of stroke. Among patients with atrial fibrillation and one or more risk factors for ischemic stroke, the recently published BRIDGE trial found forgoing bridging during interruption to be, on average, noninferior to bridging with respect to ischemic complications, with significantly fewer hemorrhagic complications.

OBJECTIVE: We sought to examine the benefits and harms of bridging anticoagulation across the spectrum of ischemic and hemorrhagic stroke risk and thereby enable more nuanced, risk-stratified decision-making when bridging is considered during initiation or interruption of vitamin $\mathrm{K}$ antagonists.

DESIGN: A Monte Carlo simulation, using a combination of literature-derived estimates, registry data, and trial data.

MAIN MEASURES: Net clinical benefit, weighting for ischemic strokes, intracranial hemorrhages, and extracranial major hemorrhages.

KEY RESULTS: The benefits and harms of bridging anticoagulation vary according to underlying patient risk profiles for both thromboembolic stroke and major intracranial bleeding. Patients at high risk of ischemic stroke and low risk of hemorrhage derive benefit from bridging during initiation or interruption of warfarin therapy. $\mathrm{Pa}-$ tients at similarly high or low risk of both outcomes may receive benefit from bridging during initiation and bridging during interruption, but this was sensitive to underlying assumptions. The need for stratification along both axes of risk was robust to a wide range of parameters.

CONCLUSIONS: Bridging anticoagulation may provide benefit to patients at high risk of ischemic stroke and low risk of intracranial hemorrhage who are initiating or interrupting warfarin therapy, while patients at high or low risk of both complications may be harmed. The use of bridging anticoagulation in patients with non-valvular atrial fibrillation should be considered only after stratification by risk of ischemic and hemorrhagic complications.

Electronic supplementary material The online version of this article (doi:10.1007/s11606-016-3932-7) contains supplementary material, which is available to authorized users.

Received May 19, 2016

Accepted November 9, 2016

Published online January 24, 2017
KEY WORDS: Monte Carlo Method; models; statistical; atrial fibrillation; thromboembolism; anticoagulants.

$\mathrm{J}$ Gen Intern Med 32(4):464-70

DOI: $10.1007 / \mathrm{s} 11606-016-3932-7$

(C) Society of General Internal Medicine 2016

\section{INTRODUCTION}

Patients with atrial fibrillation are commonly managed using vitamin $\mathrm{K}$ antagonists (VKAs), such as warfarin, to reduce the probability of stroke. When VKAs are initiated or interrupted, physicians often prescribe concomitant short-acting parenteral anticoagulants ("bridging" anticoagulants). Despite wide use of this practice, the benefit of bridging anticoagulation in both initiation and interruption remains unclear. Based on expert opinion, current guidelines recommend bridging anticoagulation for patients at high risk of stroke during VKA interruption. ${ }^{1,2}$ The recently published BRIDGE trial concluded that forgoing bridging during interruption for patients with atrial fibrillation whose $\mathrm{CHADS}_{2}$ score is 1 or greater was noninferior to bridging with regard to arterial thromboembolism, with lower rates of bleeding complications. $^{3}$

Still, several questions remain. First, the BRIDGE trial did not address the question of bridging during initiation of VKAs. More importantly, because the underlying risk of stroke varies significantly among patients who meet the BRIDGE trial's entry criteria $\left(\mathrm{CHADS}_{2}\right.$ of 1 or greater), we anticipate heterogeneity of treatment effect. Only 58 of the 1884 subjects enrolled in the BRIDGE trial had a $\mathrm{CHADS}_{2}$ score of 5 or $6 .^{3}$ In theory, though, it is these highest-risk patients who are the most likely to receive net benefit from bridging and are thus the patients recommended for bridging during interruption by most guidelines. ${ }^{1,2}$ Furthermore, intracranial hemorrhage, the most severe and disabling complication of bridging anticoagulation, is sufficiently rare that it likely represented very few, if any, of the 41 major bleeding episodes observed in that trial. ${ }^{3}$ Thus, the benefit of bridging patients with atrial fibrillation at high risk of stroke remains unclear, as does the balance of risk and benefit across the continua of ischemic and hemorrhagic risk.

Using simulation techniques, we sought to define the net clinical impact of bridging anticoagulation among patients with nonvalvular atrial fibrillation whose warfarin 
is interrupted or newly initiated. We also sought to define subpopulations for whom bridging might offer appreciable benefit or harm. We hypothesized that bridging anticoagulation could lead to clinically important net benefit or harm among patients being initiated on or interrupting VKAs depending upon the underlying risks of stroke, the underlying risk of intracranial hemorrhage, and the weighting accorded to the two outcomes.

\section{METHODS}

We created a Monte Carlo simulation model to predict the effect of bridging anticoagulation plus warfarin compared to warfarin alone on ischemic strokes, intracranial hemorrhages, and major spontaneous extracranial hemorrhages. The model assessed two situations: (1) bridging during interruption of established warfarin therapy and (2) bridging during initiation of warfarin therapy. First, we estimated the underlying daily risks of each outcome, using the two most common risk stratification scores for stroke $\left(\mathrm{CHADS}_{2}\right.$ and $\mathrm{CHA}_{2} \mathrm{DS}_{2}-$ VASc) and hemorrhages (HAS-BLED and HEMORR $2_{2}^{-}$ HAGES). ${ }^{4-8}$ Next, we simulated INRs for each day after initiation or interruption of warfarin and calculated the odds ratios for stroke and hemorrhage as a function of the patient's INR. Using those estimates (underlying daily risks, INR on each day, and odds ratios as a function of INR), we calculated the probability of each outcome with warfarin alone. We then repeated our simulation for warfarin with bridging, calculating the difference between the hypothetical cohorts with and without bridging. Because intracranial hemorrhages often have greater impact on morbidity and mortality compared with ischemic stroke, we used previously described methods to weight the two outcomes and estimate net clinical benefit. ${ }^{9,10}$ Because procedure-specific risks vary widely (a dental procedure and joint replacement would have widely different ischemic and hemorrhagic complication risks, for example), we modeled only spontaneous bleeding or ischemic events, not procedure-specific events. The model structure described here is shown schematically in Fig. 1. To ensure stable estimates, over 1 million patients were simulated for each condition. We implemented our algorithm in version 13 of STATA (College Station, TX). Code is available on request from the corresponding author.

\section{SOURCES OF MODEL PROBABILITIES}

\section{Baseline Risks of Stroke and Hemorrhage}

To estimate the underlying risks of intracranial and major extracranial hemorrhage and ischemic stroke, we sought published data that included distributions of a common stroke risk prediction tool (either $\mathrm{CHADS}_{2}$ or $\mathrm{CHA}_{2} \mathrm{DS}_{2}-\mathrm{VASc}$ ) and a common hemorrhage risk prediction tool (either HEMOR$\mathrm{R}_{2}$ HAGES or HAS-BLED). We searched the MEDLINE database using the strategy detailed in the Supplementary Appendix and reviewed the resulting papers for annual risk estimates of ischemic stroke, intracranial hemorrhage, and non-intracranial major hemorrhage. We derived daily risks of ischemic stroke, intracranial hemorrhage, and non-intracranial major hemorrhage from Friberg and colleagues' analysis of Swedish registry data, which includes information on each of the stroke and bleeding risk scores. ${ }^{5,10}$ Because intracranial hemorrhage was rare, we used regression to smooth the predicted rate of intracranial hemorrhage as a function of each bleeding risk score.

Using these underlying risk distributions, we simulated cohorts of patients with each combination of stroke risk score and hemorrhage score. Because no estimates of intracranial hemorrhage risk could be identified with the ATRIA bleeding risk score, no simulations were performed using that risk prediction tool. ${ }^{11,12}$

\section{Daily Change in INR}

In our simulation of bridging during initiation, we assumed that all patients began day zero with an INR of 1.0. Using patient-level INR data from the Michigan Anticoagulation Quality Improvement Initiative (MAQI ${ }^{2}$, a consortium of six diverse anticoagulation clinics across the state of Michigan sponsored by Blue Cross/Blue Shield), we sampled trajectories of INR as described in the Supplementary Appendix. ${ }^{13}$ These data permitted simulation under "real-world" conditions of warfarin initiation and also allowed individual-level simulation. To estimate benefit when optimal initiation protocols were used, we fitted distributions of INR to those published from the COAG trial's clinically guided dosing group. ${ }^{14}$

In our simulation of bridging during interruption, patients began day zero with a baseline INR derived from a cohort of anticoagulated atrial fibrillation patients. ${ }^{15}$ We generated declining INR values for days 1 through 5 , assuming that each patient's initial INR decayed in keeping with rates published by White and colleagues. ${ }^{16}$ We assumed that all patients resumed warfarin on day 5 and that subsequent INRs increased as they did for initiation. In our base case analysis, patients thus received bridging during interruption for 10 days total ( 5 days before and 5 days after the procedure).

\section{Odds of Stroke and Hemorrhage Conditioned on INR}

Based on data published from the ATRIA cohort, we derived ischemic and hemorrhagic event odds ratios as functions of INR. ${ }^{17} \mathrm{~A}$ detailed description of these derivations can be found in the Supplementary Appendix. We then calculated odds ratios for ischemic stroke, intracranial hemorrhage, and non-intracranial major hemorrhage for each day after initiation of warfarin. We conducted our simulation for initiation and interruption to predict the probability of each outcome with warfarin alone. 


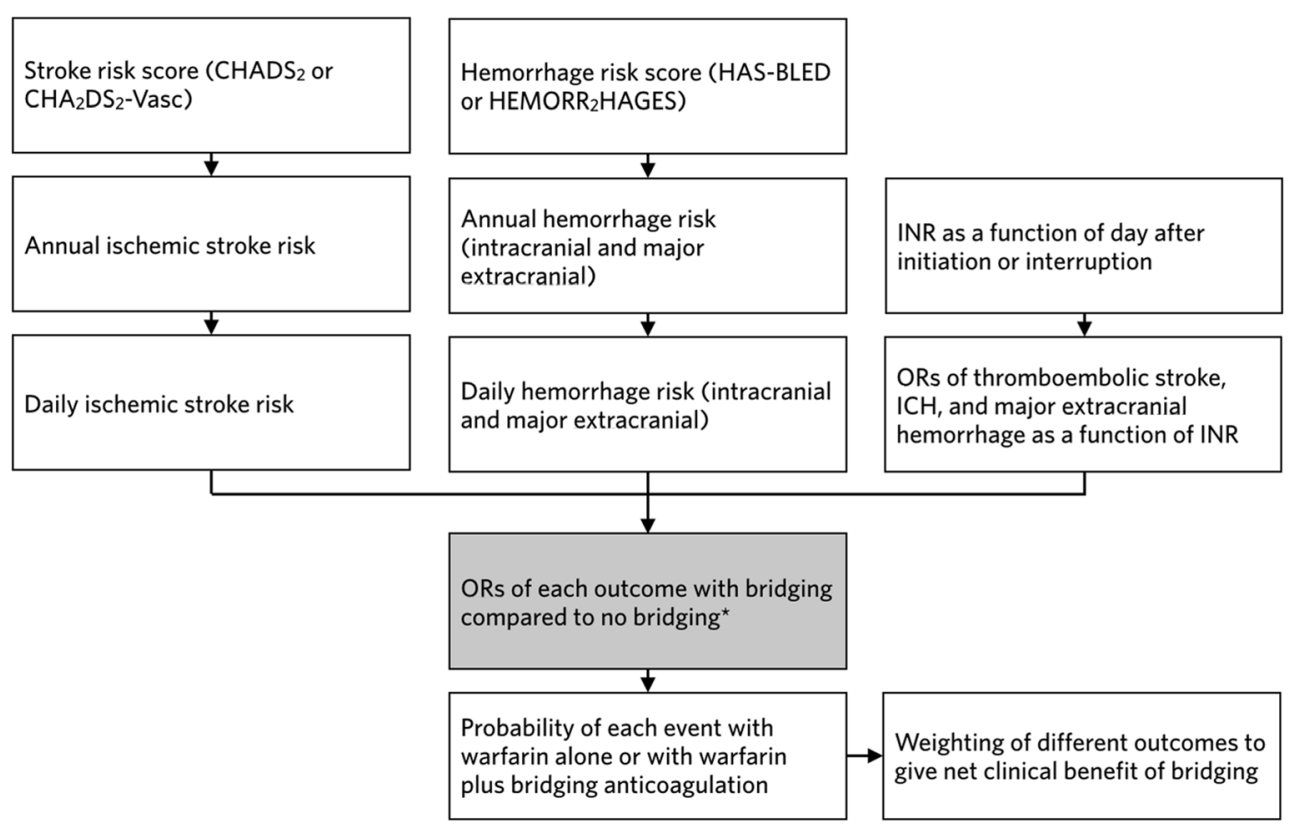

*: Running the simulation with and without this component allows an estimate of the effect of bridging.

Fig. 1 Schematic modeling diagram

To determine the relative risk of bleeding events when bridging anticoagulation is added, we applied the relative risk of hemorrhage from the BRIDGE trial. ${ }^{3}$ In separate sensitivity analyses, we substituted estimates from Siegal and colleagues' systematic review and meta-analysis of peri-procedural heparin. ${ }^{18}$ However, we found the efficacy estimates of parenteral heparin (odds ratio of 0.8) and therapeutic-range warfarin $(0.14)$ to be implausibly divergent. ${ }^{17,18}$ Our base case analysis therefore assumed bridging would be as effective as therapeutic-range warfarin for preventing ischemic events. While this assumption is driven by theory rather than data, it appeared to us the most reasonable a priori hypothesis regarding the effectiveness of bridging and the prevailing belief among clinicians. Note that this approach leads to significantly higher estimates of the benefit of bridging than would using the estimate from Siegal and colleagues' meta-analysis, as does using the relative risk found in the BRIDGE trial rather than the odds ratio estimated by Siegal and colleagues. Descriptive statistics and key references regarding relevant distributions are given in Table 1.

\section{Net Clinical Impact of Bridging}

To estimate the net clinical impact of bridging, we adopted the approach described by Singer and colleagues, assuming a relative weight of intracranial hemorrhage of 1.5 compared to ischemic stroke in our base case analyses (that is, an intracranial hemorrhage is, on average, 1.5 times worse than an ischemic stroke), with sensitivity analyses using weights from 1.0 to $2.0 .^{9}$ Because extracranial hemorrhage carries lower mortality and long-term morbidity than intracranial hemorrhage, we did not include extracranial hemorrhages in our base case estimates of net clinical benefits.

Based on our literature review, the existence of a hypercoagulable state during initiation of warfarin appeared controversial. Clinical data support such a period (with higher observed stroke rates than those predicted by risk scores), while some biochemical data suggest otherwise. ${ }^{19,20}$ Accordingly, we carried out our simulation with and without the assumption of an explicit hypercoagulable state by including or omitting a rate ratio for stroke for each day after initiation of warfarin. This was obtained from Azoulay and colleagues' cubic spline of adjusted rate ratio of ischemic stroke according to day after initiation of warfarin, provided by the corresponding author. ${ }^{19}$

We then estimated, using published data regarding the covariation of stroke and hemorrhage risk scores, the percentage of patients with non-valvular atrial fibrillation who would derive net benefit from bridging anticoagulation during initiation and interruption. ${ }^{21}$

\section{Duration of Bridging}

Our simulations of bridging during initiation used a base case time period of 7 days. Because the time to therapeutic range is not known a priori, a prescription for a bridging anticoagulant is provided to patients when warfarin is initiated; 1 week seemed to us a common clinical practice. We also simulated a 5-day period of bridging during initiation. Our simulations of interruption assumed that all patients stop warfarin 5 days prior to a procedure, resumed warfarin the day of the procedure, and received bridging for the 10 days following interruption of warfarin ( 5 days before and 5 days after their procedure). 
Table 1 Characteristics of Modeled Distributions of Key Variables

\begin{tabular}{|c|c|c|c|c|}
\hline Parameter & Base case estimate & $\begin{array}{l}\text { Distribution }(95 \% \\
\text { CI) }\end{array}$ & Sensitivity analyses* & References \\
\hline Annual incidence of ischemic stroke & $0.6 \%$ to $14.6 \%$ & $\begin{array}{l}\text { Fixed for each } \\
\text { CHADS }_{2} \text { score }\end{array}$ & $\begin{array}{l}\text { Fixed for each } \mathrm{CHA}_{2} \mathrm{DS}_{2} \text {-Vasc score }(0.2 \% \\
\text { to } 14.4 \%)\end{array}$ & 5,10 \\
\hline $\mathrm{CHADS}_{2}$ score $=0$ & $0.6 \%$ & - & 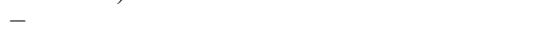 & \\
\hline $\mathrm{CHADS}_{2}$ score $=1$ & $3.4 \%$ & - & - & \\
\hline $\mathrm{CHADS}_{2}$ score $=2$ & $4.7 \%$ & - & - & \\
\hline $\mathrm{CHADS}_{2}$ score $=3$ & $8.0 \%$ & - & - & \\
\hline $\mathrm{CHADS}_{2}$ score $=4$ & $12.6 \%$ & - & - & \\
\hline $\mathrm{CHADS}_{2}$ score $=5$ & $14.1 \%$ & - & - & \\
\hline $\mathrm{CHADS}_{2}$ score $=6$ & $14.6 \%$ & - & - & \\
\hline $\begin{array}{l}\text { Annual incidence of intracranial } \\
\text { hemorrhage }\end{array}$ & $0.1 \%$ to $1.3 \%$ & $\begin{array}{l}\text { Fixed for each } \\
\text { HAS-BLED score }\end{array}$ & $\begin{array}{l}\text { Rates fixed by HAS-BLED score without } \\
\text { regression smoothing (below); fixed for each } \\
\text { HEMORR }_{2} \text { HAGES score }(0.1 \% \text { to } 4 \% \text { ) }\end{array}$ & 5,10 \\
\hline HAS-BLED score $=0$ & $0.1 \%$ & - & $0.1 \%$ & \\
\hline HAS-BLED score $=1$ & $0.5 \%$ & - & $0.5 \%$ & \\
\hline HAS-BLED score $=2$ & $0.9 \%$ & - & $0.8 \%$ & \\
\hline HAS-BLED score $=3$ & $1.1 \%$ & _ & $1.4 \%$ & \\
\hline HAS-BLED score $=4$ & $1.3 \%$ & _- & $1.1 \%$ & \\
\hline HAS-BLED score $=5$ & $1.3 \%$ & - & $1.2 \%$ & \\
\hline HAS-BLED score $=6$ & $1.3 \%$ & - & $1.4 \%$ & \\
\hline $\begin{array}{l}\text { Annual incidence of extracranial } \\
\text { major hemorrhage }\end{array}$ & $0.5 \%$ to $14.5 \%$ & $\begin{array}{l}\text { Fixed for each } \\
\text { HAS-BLED score }\end{array}$ & $\begin{array}{l}\text { Fixed for each HEMORR }{ }_{2} \text { HAGES score } \\
(0.4 \% \text { to } 21.1 \%)\end{array}$ & 5,10 \\
\hline HAS-BLED score $=0$ & $0.5 \%$ & - & - & \\
\hline HAS-BLED score $=1$ & $2.1 \%$ & - & - & \\
\hline HAS-BLED score $=2$ & $3.6 \%$ & - & - & \\
\hline HAS-BLED score $=3$ & $5.5 \%$ & - & - & \\
\hline HAS-BLED score $=4$ & $7.8 \%$ & - & - & \\
\hline HAS-BLED score $=5$ & $9.0 \%$ & _ & - & \\
\hline HAS-BLED score $=6$ & $14.5 \%$ & - & _- & \\
\hline $\begin{array}{l}\text { Odds ratio for ischemic stroke with } \\
\text { bridging, base case }\end{array}$ & 0.14 & Normal (0.11-0.17) & $0.74(0.47-0.97)^{\dagger}$ & 17,18 \\
\hline $\begin{array}{l}\text { Relative risk for hemorrhagic events } \\
\text { with bridging }\end{array}$ & 3.86 & $\begin{array}{l}\text { Normal }(1.50- \\
6.59)\end{array}$ & Odds ratio $3.60(1.87-3.01)$ & 3 \\
\hline $\begin{array}{l}\text { Relative weight of intracranial } \\
\text { hemorrhage compared with ischemic } \\
\text { stroke (to estimate net clinical } \\
\text { benefit) }\end{array}$ & 1.5 & Fixed & $1.0,1.5$, or 2.0 & 9 \\
\hline $\begin{array}{l}\text { Starting INR for interruption } \\
\text { simulations }\end{array}$ & 2.42 & $\begin{array}{l}\text { Normal, and } \\
\text { limited to } \geq 1.0 \\
(1.09-3.01)\end{array}$ & - & 15 \\
\hline Duration of bridging during initiation & 7 days & Fixed & 5 or 10 days & Assumed \\
\hline $\begin{array}{l}\text { Duration of bridging during } \\
\text { interruption }\end{array}$ & 10 days & Fixed & 7 or 14 days & Assumed \\
\hline $\begin{array}{l}\text { Relative weight of extracranial major } \\
\text { hemorrhage compared with ischemic } \\
\text { stroke }\end{array}$ & 0 & Fixed & 0.01 & Assumed \\
\hline $\begin{array}{l}\text { Trajectories of INR after initiation or } \\
\text { resumption of warfarin }\end{array}$ & $\begin{array}{l}\text { Sampled per patient } \\
\text { from MAQI }{ }^{2} \text { registry }\end{array}$ & - & Sampled from COAG trial data & 13,14 \\
\hline $\begin{array}{l}\text { Relative risk of ischemic stroke } \\
\text { during initiation (a hypercoagulable } \\
\text { state) }\end{array}$ & $\begin{array}{l}1 \text { (no hypercoagulable } \\
\text { state) }\end{array}$ & - & $\begin{array}{l}\text { Varies by day after initiation; normal on each } \\
\text { day }\end{array}$ & 19 \\
\hline Day 1 & - & - & $1.65(1.28-2.13)$ & \\
\hline Day 2 & - & - & $2.18(1.46-3.25)$ & \\
\hline Day 3 & _ & _ & $2.33(1.50-3.62)$ & \\
\hline Day 4 & - & - & $2.14(1.39-3.29)$ & \\
\hline Day 5 & - & - & $1.80(1.18-2.73)$ & \\
\hline Day 6 & - & - & $1.46(0.94-2.28)$ & \\
\hline Day 7 & - & - & $1.22(0.75-2.00)$ & \\
\hline
\end{tabular}

* Some authors would refer to these as scenario analyses rather than sensitivity analyses. We here use the more general and more widely understood term

${ }^{\prime}$ As described in the text, these two estimates are discordant, with non-overlapping confidence intervals, and produce meaningfully different results

\section{SENSITIVITY ANALYSES}

In one- and two-way sensitivity analyses, we varied the risk scores used, whether regression was used to smooth rates of hemorrhagic outcomes, the relative impact of bridging on the risks of ischemic stroke and hemorrhagic events (from either the BRIDGE trial or a meta-analysis that included larger numbers of patients), the relative weight given to each major outcome (ischemic stroke and intracranial hemorrhage), the duration of bridging, whether a small disutility was accorded to extracranial major hemorrhages, the origin of our INR trajectories, and whether a clinically significant hypercoagulable state exists on initiation of warfarin. Sensitivity analyses are summarized in Table 1 . Those that materially changed our conclusions are discussed below; others are presented in the Supplementary Appendix. 
Table 2 Estimated Net Clinical Benefit from 10 Days of Bridging During Interruption for Patients with Different Risk Scores For Ischemic Stroke $\left(\mathrm{CHADS}_{2}\right)$ and Hemorrhagic Complications (HAS-BLED) and Increase in Spontaneous Extracranial Major Hemorrhages at each HASBLED Score

\begin{tabular}{|c|c|c|c|c|c|c|c|c|}
\hline \multirow[b]{3}{*}{ HAS-BLED } & \multicolumn{7}{|c|}{$\begin{array}{l}\text { Change in weighted strokes per } 100,000, \text { ICH } 1.5 \text { times worse } \\
\text { than ischemic stroke* }\end{array}$} & \multirow[t]{3}{*}{ Episodes of extracranial major bleeding, per $100,000 \dagger$} \\
\hline & \multicolumn{7}{|c|}{ CHADS2 } & \\
\hline & 0 & 1 & 2 & 3 & 4 & 5 & 6 & \\
\hline 0 & -2 & -43 & -62 & -114 & -188 & $\mathrm{~N} / \mathrm{P}$ & $\mathrm{N} / \mathrm{P}$ & 34 \\
\hline 1 & 28 & -14 & -33 & -84 & -158 & -183 & $\mathrm{~N} / \mathrm{P}$ & 61 \\
\hline 2 & 51 & 10 & -10 & -61 & -135 & -160 & -169 & 114 \\
\hline 3 & 68 & 27 & 7 & -44 & -119 & -144 & -152 & 194 \\
\hline 4 & 79 & 37 & 18 & -33 & -107 & -133 & -141 & 301 \\
\hline 5 & 83 & 42 & 23 & -28 & -102 & -128 & -136 & 437 \\
\hline 6 & 81 & 40 & 20 & -31 & -103 & -130 & -138 & 606 \\
\hline
\end{tabular}

*An intracranial hemorrhage is weighted to be 1.5 times clinically worse than an ischemic stroke

tEpisodes of spontaneous extracranial major bleeding induced by bridging during interruption

Model estimation of stroke and bleeding outcomes after bridging anticoagulation for 5 days before and 5 days after a procedure in patients whose warfarin is interrupted in patients with a range of CHADS2 risk scores for ischemic stroke (columns) and HAS-BLED risk scores for hemorrhagic complications (rows). Shaded cells indicate a net benefit in weighted strokes. "N/P" indicates combinations of scores that are not possible, given clinical conditions that are included in both scores. For example, a patient with a CHADS, score of 6 must be at least 75 years old and have a history of hypertension. Because both are also included in HAS-BLED, that patient must have a HAS-BLED score of at least 2. The number in each cell indicates the weighted strokes caused or prevented for that combination of risk scores. For example, bridging 100,000 patients with CHADS 2 and HAS-BLED scores of 0 for 10 days during warfarin interruption would avert 2 weighted net strokes, on average, and induce 34 extracranial major hemorrhages

\section{RESULTS}

In our base case simulations of bridging during initiation and bridging during interruption, between $47 \%$ and $80 \%$ of patients would receive net benefit, depending on underlying assumptions. The results of our base case simulation of bridging during interruption are shown in Tables 2 and 3. Table 2 shows the change in weighted strokes (intracranial hemorrhage is weighted as 1.5 times ischemic stroke to reflect the generally worse outcomes of that event) and change in extracranial major hemorrhages when bridging during interruption of warfarin. Similarly, Table 3 shows the results of our base case simulation of bridging during initiation, with change in weighted strokes and change in extracranial major hemorrhages stratified by risk scores. Each cell in Tables 2 and 3 contains the net change in weighted strokes per 100,000 bridged patients, relative to warfarin alone, for that combination of stroke risk scores and bleeding risk scores. Negative numbers represent reduction in stroke (net benefit), while positive numbers represent an increase in weighted strokes (a net harm).

We found significant variation in the net benefit of bridging based on patients' underlying risks of ischemic and hemorrhagic stroke. In both initiation and interruption of warfarin, patients at high risk of stroke and low risk of intracranial hemorrhage stand to benefit from bridging anticoagulation. For example, bridging 100,000 patients with a $\mathrm{CHADS}_{2}$ score

Table 3 Estimated Net Clinical Benefit from 7 Days of Bridging During Initiation for Patients with Different Risk Scores for Ischemic Stroke $\left(\mathrm{CHADS}_{2}\right)$ and Hemorrhagic Complications (HAS-BLED) and Increase in Spontaneous Extracranial Major Hemorrhages At Each HASBLED Score

\begin{tabular}{|c|c|c|c|c|c|c|c|c|}
\hline \multirow[b]{3}{*}{ HAS-BLED } & \multicolumn{7}{|c|}{$\begin{array}{l}\text { Change in weighted strokes per } 100,000, \text { ICH } 1.5 \text { times worse } \\
\text { than ischemic stroke* }\end{array}$} & \multirow[t]{3}{*}{ Episodes of extracranial major bleeding, per $100,000 \uparrow$} \\
\hline & \multicolumn{7}{|c|}{ CHADS2 } & \\
\hline & 0 & 1 & 2 & 3 & 4 & 5 & 6 & \\
\hline 0 & -4 & -47 & -67 & -120 & -197 & $\mathrm{~N} / \mathrm{P}$ & $\mathrm{N} / \mathrm{P}$ & 23 \\
\hline 1 & 16 & -26 & -47 & -100 & -176 & -203 & $\mathrm{~N} / \mathrm{P}$ & 43 \\
\hline 2 & 33 & -10 & -31 & -83 & -161 & -186 & -194 & 80 \\
\hline 3 & 44 & 2 & -19 & -72 & -148 & -174 & -184 & 135 \\
\hline 4 & 52 & 9 & -11 & -64 & -140 & -167 & -175 & 210 \\
\hline 5 & $5 \overline{5}$ & 13 & -8 & -61 & -138 & -163 & -172 & 305 \\
\hline 6 & 54 & 11 & -9 & -62 & -139 & -164 & $-17 \overline{3}$ & 422 \\
\hline
\end{tabular}

*An intracranial hemorrhage is weighted to be 1.5 times clinically worse than an ischemic stroke

tEpisodes of spontaneous extracranial major bleeding induced by bridging during interruption

Model estimation of stroke and bleeding outcomes after 7 days of bridging during initiation in patients with a range of CHADS, risk scores for ischemic stroke (columns) and HAS-BLED risk scores for hemorrhagic complications (rows). Shaded cells indicate a net benefit in weighted strokes. The number in each cell indicates the weighted strokes caused or prevented for that combination of risk scores. For example, bridging 100,000 patients with CHADS2 and HAS-BLED scores of 0 for 7 days during warfarin initiation would avert 4 weighted net strokes, on average, and induce 23 extracranial major hemorrhages

"N/P" indicates combinations of scores that are not possible, given clinical conditions that are included in both scores. For example, a patient with a $\mathrm{CHADS}_{2}$ score of 6 must be at least 75 years old and have a history of hypertension. Because "Age $>65$ " and "Hypertension history" are also included in HAS-BLED, that patient must have a HAS-BLED score of at least 2 
of 5 and a HAS-BLED score of 1 during an interruption of warfarin would avert approximately 219 ischemic strokes, but cause approximately 24 intracranial bleeding events. When those outcomes are weighted as in our base case analysis, bridging during interruption would lead to a net clinical benefit of 183 weighted strokes prevented per 100,000 patients (shown in Table 2).

Overall, net clinical benefit appeared to be most sensitive to the weighting given to intracranial hemorrhage relative to ischemic stroke. Because extracranial hemorrhages are not directly comparable to strokes and the small disutility we assigned led to few changes in the decision to bridge, extracranial major hemorrhages are reported separately.

Two sensitivity analyses produced notable results. When we assumed that there is a significant hypercoagulable state with initiation of warfarin, between 79 and $90 \%$ of patients derive net benefit from bridging during initiation compared with initiation of warfarin alone. In contrast, when we assumed that bridging is as effective as reported by Siegal and colleagues (RR of 0.8 for ischemic stroke), rather than assuming bridging is as effective as therapeutic-range warfarin ( $R R$ of 0.14), no simulated groups, in either bridging during initiation or bridging during interruption, received benefit. Additional tables, with outcomes according to $\mathrm{CHA}_{2} \mathrm{DS}_{2}$-VASc and HEMORR 2 -HAGES scores, along with tables for different sensitivity analyses, are included in the Supplementary Appendix. While variations in each parameter led to different magnitudes of predicted benefit or harm, the need for stratification by both gradients of risk was consistent.

\section{DISCUSSION}

Bridging anticoagulation is often prescribed for patients with atrial fibrillation when VKAs are initiated or interrupted, based on expert-consensus guidelines. In contrast to the recently reported BRIDGE trial, the summary results of which would suggest forgoing bridging entirely, our simulations suggest that subpopulations may receive benefit from bridging. In fact, in our base case simulation of bridging during interruption, a majority of patients stand to benefit, and in almost all sensitivity analyses, patients at high risk of ischemic complications and low risk of hemorrhagic complications receive benefit.

In this simulation-based analysis, the net clinical benefit of bridging varies substantially based on underlying risk of ischemic and hemorrhagic complications, ranging from (in our base case simulation of bridging during interruption) 83 weighted strokes caused to 188 weighted strokes averted per 100,000 patients bridged. Our analysis demonstrates that, for this practice to offer benefit to patients, the decision to prescribe bridging anticoagulation must be personalized on predicted risk of ischemic and hemorrhagic complications. We have included tables (here and in the Supplementary Appendix) under many different assumptions to allow more personalized decisions regarding bridging during initiation and bridging during interruption for patients with non-valvular atrial fibrillation. Note that we do not here consider procedure-specific risks or benefits, as these vary widely; such risks must be considered in addition to the spontaneous risks we consider here.

There appears to be a subset of patients who would derive clinically meaningful benefit from bridging anticoagulation. For example, prescribing a 7-day bridge to 100,000 patients with a $\mathrm{CHA}_{2} \mathrm{DS}_{2}$-VASc score of 5 and a HAS-BLED score of 0 initiating warfarin would prevent around 81 ischemic strokes at the expense of 3 intracranial hemorrhages and 24 extracranial major hemorrhages caused. This benefit turns to harm, though, when bleeding risk increases. A 100,000-person cohort of patients with a $\mathrm{CHA}_{2} \mathrm{DS}_{2}$-VASc score of 5 and a HASBLED score of 5 would stand to receive a more modest benefit and perhaps a harm: around 81 ischemic strokes would be prevented, at the expense of 43 hemorrhagic strokes and 310 extracranial major hemorrhages caused.

Our analysis is subject to several important limitations. First, we are dependent upon literature-derived risks, which in turn depend on the predictive ability of the risk scores used. To our knowledge, neither HAS-BLED nor HEMORR $2_{2}$ HAGES have been prospectively validated to predict intracranial hemorrhage. Prediction of ICH is challenging, and even in the large Swedish registry non-monotonic changes in risk by score (for example, the highest risk of intracranial hemorrhage was seen at a HAS-BLED score of 3) leave us with some concern about the accuracy of these prediction scores for this outcome. Second, our risk distributions are derived from a Scandinavian registry of patients with previously diagnosed atrial fibrillation; scores in this and other registries are likely to be systematically higher than in patients with newly diagnosed atrial fibrillation and patients being initiated on VKAs, and they may have systematic differences compared with patients in other countries or regions. Third, the ability to translate careful risk stratification into practice is hampered by inclusion of parameters that may not be available when initiating anticoagulation, such as labile INR (in HAS-BLED) or genetic predisposition to hemorrhage (in HEMORR 2 -HAGES). Fourth, these results do not apply to patient groups for whom immediate anticoagulation is desired (such as patients with pulmonary emboli). Fifth, we modeled net clinical benefit, not cost-effectiveness. The small benefits predicted in our simulation may not be clinically meaningful or cost-effective for all groups. Future cost-effectiveness analyses could better define what subgroups of patients receive adequate benefit to justify the cost of bridging. This method also leaves out some clinically significant events, such as skin necrosis and thrombocytopenia. While the disutility of such outcomes is likely much lower than the disutility of long-term disability from stroke, they remain clinically significant events. And finally, we are not aware of an outcomes database of sufficient size and detail to validate our predicted results; accordingly, the predictive fit of our model cannot currently be assessed.

Despite these limitations, our analysis provides additional guidance on the use of bridging anticoagulation 
among atrial fibrillation patients being initiated on warfarin or whose warfarin must be interrupted. The BRIDGE trial concluded that forgoing bridging is non-inferior by more than $0.1 \%$ compared to bridging during interruption for patients with atrial fibrillation and a $\mathrm{CHADS}_{2}$ score of 1 or greater; our analysis adds that patients at highest risk of ischemic complications, who were underrepresented in the BRIDGE trial, stand to receive benefit from bridging during both initiation and interruption. Our simulations further suggest that bridging anticoagulation should be used only after careful risk stratification and consideration of the modest benefit conferred. If guidelines are to recommend bridging anticoagulation for patients with atrial fibrillation initiating or interrupting VKAs, stratification by both risk of ischemic and hemorrhagic stroke is essential.

Acknowledgements: The authors wish to thank Dr. Samy Suissa for providing day-specific rate ratios from their investigation of the hypercoagulable state in warfarin initiation, Drs. Margaret Fang, Anita Misra-Hebert, and Michael Rothberg for their helpful comments on earlier drafts of this manuscript, and the Ann Arbor VA for fellowship support.

Corresponding Author: Matthew A. Pappas, MD, MPH; Center for Value-Based Care Research, Medicine Institute, Cleveland Clinic, 9500 Euclid Ave, M2 Annex, Cleveland, OH 44195, USA (e-mail: pappasm@umich.edu).

\section{Compliance with ethical standards:}

Funding Sources: The authors received no specific funding for this work. The primary author received fellowship support from the Ann Arbor VA.

Conflict of Interest: The authors declare that they do not have a conflict of interest.

\section{REFERENCES}

1. Douketis JD, Spyropoulos AC, Spencer FA, et al. Perioperative management of antithrombotic therapy: antithrombotic therapy and prevention of thrombosis, 9th ed: American College of Chest Physicians Evidence-Based Clinical Practice Guidelines. Chest. 2012;141(2 Suppl):e326S-50. doi:10.1378/chest.11-2298.

2. January CT, Wann LS, Alpert JS, et al. 2014 AHA/ACC/HRS guideline for the management of patients with atrial fibrillation: executive summary: a report of the American College of Cardiology/American Heart Association Task Force on Practice Guidelines and the Heart Rhythm Society. J Am Coll Cardiol. 2014;64(21):2246-80. doi:10.1016/j.jacc. 2014.03.021.

3. Douketis JD, Spyropoulos AC, Kaatz S, et al. Perioperative bridging anticoagulation in patients with atrial fibrillation. N Engl J Med. 2015. doi: 10.1056/NEJMoa1501035.

4. Gage BF, Waterman AD, Shannon W, Boechler M, Rich MW, Radford MJ. Validation of clinical classification schemes for predicting stroke: results from the National Registry of Atrial Fibrillation. JAMA. 2001;285(22):2864-70.

5. Friberg L, Rosenqvist M, Lip GYH. Evaluation of risk stratification schemes for ischaemic stroke and bleeding in 182678 patients with atrial fibrillation: the Swedish Atrial Fibrillation cohort study. Eur Heart J. 2012;33(12):1500-10. doi:10.1093/eurheartj/ehr488.

6. Lip GYH, Nieuwlaat R, Pisters R, Lane DA, Crijns HJGM. Refining clinical risk stratification for predicting stroke and thromboembolism in atrial fibrillation using a novel risk factor-based approach: the euro heart survey on atrial fibrillation. Chest. 2010;137(2):263-72. doi:10.1378/ chest.09-1584.

7. Gage BF, Yan Y, Milligan PE, et al. Clinical classification schemes for predicting hemorrhage: results from the National Registry of Atrial Fibrillation (NRAF). Am Heart J. 2006;151(3):713-9. doi:10.1016/j.ahj. 2005.04.017.

8. Pisters R, Lane DA, Nieuwlaat R, de Vos CB, Crijns HJGM, Lip GYH. A novel user-friendly score (HAS-BLED) to assess 1-year risk of major bleeding in patients with atrial fibrillation: the Euro Heart Survey. Chest. 2010;138(5):1093-100. doi:10.1378/chest.10-0134.

9. Singer DE, Chang Y, Fang MC, et al. The net clinical benefit of warfarin anticoagulation in atrial fibrillation. Ann Intern Med. 2009;151(5):297305.

10. Friberg L, Rosenqvist M, Lip GYH. Net clinical benefit of warfarin in patients with atrial fibrillation: a report from the Swedish atrial fibrillation cohort study. Circulation. 2012;125(19):2298-307. doi:10.1161/ CIRCULATIONAHA. 111.055079.

11. Fang MC, Go AS, Chang $\mathbf{Y}$, et al. A new risk scheme to predict warfarinassociated hemorrhage: The ATRIA (Anticoagulation and Risk Factors in Atrial Fibrillation) Study. J Am Coll Cardiol. 2011;58(4):395-401. doi:10. 1016/j.jacc.2011.03.031.

12. Singer DE, Chang $\mathbf{Y}$, Borowsky LH, et al. A new risk scheme to predict ischemic stroke and other thromboembolism in atrial fibrillation: the ATRIA study stroke risk score. J Am Heart Assoc. 2013;2(3):e000250. doi: 10.1161/JAHA. 113.000250.

13. Barnes GD, Gu X, Haymart B, et al. The predictive ability of the CHADS2 and CHA2DS2-VASc scores for bleeding risk in atrial fibrillation: the MAQI(2) experience. Thromb Res. 2014;134(2):294-9. doi:10.1016/j. thromres.2014.05.034.

14. Kimmel SE, French B, Kasner SE, et al. A pharmacogenetic versus a clinical algorithm for warfarin dosing. N Engl J Med. 2013;369(24):228393. doi:10.1056/NEJMoa1310669.

15. Le Heuzey J-Y, Ammentorp B, Darius H, et al. Differences among western European countries in anticoagulation management of atrial fibrillation. Data from the PREFER IN AF registry. Thromb Haemost. 2014;111(5):833-41. doi:10.1160/TH13-12-1007.

16. White RH, McKittrick T, Hutchinson R, Twitchell J. Temporary discontinuation of warfarin therapy: changes in the international normalized ratio. Ann Intern Med. 1995;122(1):40-2.

17. Singer DE, Chang Y, Fang MC, et al. Should patient characteristics influence target anticoagulation intensity for stroke prevention in nonvalvular atrial fibrillation?: The ATRIA study. Circ Cardiovasc Qual Outcomes. 2009;2(4):297-304. doi:10.1161/CIRCOUTCOMES. 108. 830232 .

18. Siegal D, Yudin J, Kaatz S, Douketis JD, Lim W, Spyropoulos AC. Periprocedural heparin bridging in patients receiving vitamin $\mathrm{K}$ antagonists: systematic review and meta-analysis of bleeding and thromboembolic rates. Circulation. 2012;126(13):1630-9. doi:10.1161/ CIRCULATIONAHA. 112.105221.

19. Azoulay L, Dell'Aniello S, Simon TA, Renoux C, Suissa S. Initiation of warfarin in patients with atrial fibrillation: early effects on ischaemic strokes. Eur Heart J. 2014;35(28):1881-7. doi:10.1093/eurheartj/ eht499.

20. Zeuthen EL, Lassen JF, Husted SE. Is there a hypercoagulable phase during initiation of antithrombotic therapy with oral anticoagulants in patients with atrial fibrillation? Thromb Res. 2003;109(5-6):241-6. doi:10.1016/S0049-3848(03)00240-8.

21. Marcucci M, Lip GYH, Nieuwlaat R, Pisters R, Crijns HJGM, Iorio A. Stroke and bleeding risk co-distribution in real-world patients with atrial fibrillation: the euro heart survey. Am J Med. 2014;127(10):979-986.e2. doi:10.1016/j.amjmed.2014.05.003. 